\title{
Estimates for Quasiconformal Mappings onto \\ Canonical Domains (II)
}

\author{
Vo Dang Thao
}

\begin{abstract}
In this paper we establish estimates for normal $K$-quasiconformal mappings $z=g(w)$ of any finitely-connected domain in the extended $w$-plane onto the interior or exterior of the unit circle or the extended $z$-plane with $n(\geq 0)$ slits on the circles $|z|=$ $R_{j} \quad(j=1, \ldots, n)$. The bounds in the estimates for $R_{j},|g(w)|$, etc. are explicitly given. They are sharp or asymptotically sharp and deduced mainly from estimates for the inverse mappings of $g$ in our previous paper [10] based on Carleman's and Grötzsch's inequalities and partly improved here. A generalization of the Schwarz lemma and improvements of some classical inequalities for conformal mappings are shown.
\end{abstract}

Keywords: K-quasiconformal mappings, Riemann moduli of a multiply-connected domain AMS subject classification: 30C62, 30C75, 30C80, 30C30, 30C35

\section{Introduction and notations}

This paper is a continuation of our previous paper [11] where estimates for $K$ quasiconformal mappings (see the definition in [4: p.16]) onto a circular $\operatorname{ring} Q<$ $|z|<1$ with some circular slits are given. Here we shall establish estimates for normal $K$-quasiconformal mappings $z=g(w)$ of any finitely-connected domain in the extended $w$-plane onto the interior or exterior of the unit circle or the extended $z$-plane with some circular slits.

Throughout this paper, we use the following notations. Let $w=f(z)$ be a $K$ quasiconformal mapping of a domain $A$ in the extended $z$-plane onto a domain $B$ in the extended $w$-plane. Put

$$
\begin{aligned}
& m(R, f)=\{\min |w|: w \in E(R, f)\} \\
& M(R, f)=\{\max |w|: w \in E(R, f)\}
\end{aligned}
$$

where $E(R, f)$ means the set of the $w$-plane corresponding to the circle $|z|=R$ by $f$, that may contain some slits or a circle as boundary components of $A$. Moreover, denote by $S(R, f)$ the inner area of the domain bounded by the above set $E(R, f)$.

Vo Dang Thao: Univ. Ho Chi Minh City, Dept. Math., 227 Nguyen Van Cu, Q 5, Ho Chi Minh City, Vietnam; vodangthao@yahoo.com 
If $A$ contains $z=0$ and $f(0)=0$, then put

$$
\begin{aligned}
& m^{\prime}(0, f)=\lim _{R \rightarrow 0} \frac{m(R, f)}{R^{\frac{1}{K}}}, \quad m^{*}(0, f)=\lim _{R \rightarrow 0} \frac{m(R, f)}{R^{K}} \\
& M^{\prime}(0, f)=\lim _{R \rightarrow 0} \frac{M(R, f)}{R^{\frac{1}{K}}}, \quad M^{*}(0, f)=\lim _{R \rightarrow 0} \frac{M(R, f)}{R^{K}} \\
& S^{\prime}(0, f)=\lim _{R \rightarrow 0} \frac{S(R, f)}{\pi R^{\frac{2}{K}}} .
\end{aligned}
$$

If $A$ contains $z=\infty$ and $f(\infty)=\infty$, then put

$$
\begin{aligned}
& m^{\prime}(\infty, f)=\lim _{R \rightarrow \infty} \frac{m(R, f)}{R^{\frac{1}{K}}}, \quad m^{*}(\infty, f)=\lim _{R \rightarrow \infty} \frac{m(R, f)}{R^{K}} \\
& M^{\prime}(\infty, f)=\lim _{R \rightarrow \infty} \frac{M(R, f)}{R^{\frac{1}{K}}}, \quad M^{*}(\infty, f)=\lim _{R \rightarrow \infty} \frac{M(R, f)}{R^{K}} \\
& S^{\prime}(\infty, f)=\lim _{R \rightarrow \infty} \frac{S(R, f)}{\pi R^{\frac{2}{K}}} .
\end{aligned}
$$

Throughout this paper, we suppose that the introduced limits exist. Clearly, if $E(R, f)$ separates the points 0 and $\infty$, then

$$
\begin{aligned}
m^{\prime}(0, f)^{2} & \leq S^{\prime}(0, f) \leq M^{\prime}(0, f)^{2} \\
m^{\prime}(\infty, f)^{2} & \leq S^{\prime}(\infty, f) \leq M^{\prime}(\infty, f)^{2}
\end{aligned}
$$

We consider now the three following classes of $K$-quasiconformal mappings onto canonical domains.

Let $B_{1}$ be any domain in the disk $|w|<1,0 \in B_{1}$, bounded by $C_{1}$ as the external boundary component with $\max \left\{|w|: w \in C_{1}\right\}=1$ and $p n \quad\left(p \in \mathbb{N}, n \in \mathbb{N}_{0}=\mathbb{N} \cup\{0\}\right)$ others $\sigma_{1}, \ldots, \sigma_{p n}$. Suppose that $B_{1}$ is transformed into itself by the rotation

$$
t=e^{i \frac{2 \pi}{p}} w
$$

Denote by

$S_{1}(\leq \pi)$ - the inner area of the domain bounded by $C_{1}$

$S\left(\leq S_{1}\right)$ - the inner area of the domain $B_{1}$

$G_{1} \quad$ the class of all $K$-quasiconformal mappings $z=g(w)$

each of which maps $B_{1}$ onto the disk $|z|<1$ that has been slit along $p n$ circular arcs $L_{1}(g), \ldots, L_{p n}(g)$ concentric with the unit circle such that $|z|=1$ and $L_{j}$ correspond to $C_{1}$ and $\sigma_{j}(j=1, \ldots, p n)$, respectively, $g(0)=0$, and satisfies the $p$-fold rotational symmetry

$$
g\left(e^{i \frac{2 \pi}{p}} w\right)=e^{i \frac{2 \pi}{p}} g(w)
$$

for all $w \in B_{1}$. Clearly, this condition is trivial for $p=1$.

Let $B_{2}$ be any domain in $|w|>1$ containing $w=\infty$ bounded by $p n$ boundary components $\sigma_{1}, \ldots, \sigma_{p n}$ and $C_{2}$ whose interior contains $|w|<1$ but cannot contain 
any disk $\left|w-w_{0}\right|<r$ with $r>1$. Suppose that $B_{2}$ is transformed into itself by rotation (1.3). Denote by

$S_{2}$ - the external area of the compact set bounded by $C_{2}$

$G_{2}$ - the class of all $K$-quasiconformal mappings $z=g(w)$

each of which maps $B_{2}$ onto the domain $|z|>1$ that has been slit along $p n$ circular $\operatorname{arcs} L_{1}(g), \ldots, L_{p n}(g)$ concentric with the unit circle such that $|z|=1$ and $L_{j}$ correspond to $C_{2}$ and $\sigma_{j}(j=1, \ldots, p n)$, respectively, $g(\infty)=\infty$, and satisfies (1.4) for all $w \in B_{2}$.

Let $B_{3}$ be any domain in the extended $w$-plane containing $w=0$ and $w=$ $\infty$ bounded by $p n$ boundary components $\sigma_{1}, \ldots, \sigma_{p n}$ and transformed into itself by rotation (1.3). Denote by $G_{3}$ the class of all $K$-quasiconformal mappings $z=g(w)$ each of which maps $B_{3}$ onto the extended $z$-plane that has been slit along $p n$ circular arcs $L_{1}(g), \ldots, L_{p n}(g)$ concentric with the unit circle such that $L_{j}$ corresponds to $\sigma_{j}(j=1, \ldots, p n), g(0)=0, g(\infty)=\infty$, and satisfies (1.4) for all $w \in B_{3}$. Moreover, suppose $m^{*}(\infty, g)=1$ for $g \in G_{3}$.

For each $\sigma_{j}$ as boundary component of $B_{\nu}$ and for each $L_{j}(g) \quad\left(g \in G_{\nu} ; j=\right.$ $1, \ldots, p n ; \nu=1,2,3)$ put

$$
\begin{aligned}
& c_{j}=\min _{w \in \sigma_{j}}|w| \\
& d_{j}=\max _{w \in \sigma_{j}}|w| \\
& R_{j}(g)=|z| \quad\left(z \in L_{j}(g)\right) \\
& R_{0}(g)=\max _{1 \leq j \leq p n} R_{j}(g) \\
& s_{0}=\min _{1 \leq j \leq p n} s_{j} \\
& s=\sum_{j=1}^{p n} s_{j}
\end{aligned}
$$

where $s_{j}$ means the external area of the compact set bounded by $\sigma_{j}$.

The principal aim of this paper is to estimate $|g(w)|$, the radii $R_{j}(g)$, etc. for $w \in B_{\nu}, g \in G_{\nu} \quad(j=1, \ldots, p n ; \nu=1,2,3)$. For $K=1$ (conformal mappings) these radii are nothing but the Riemann moduli of the domains $B_{\nu}$ (see [5: p. 334]). The obtained estimates are sharp or asymptotically sharp. Their bounds are explicitly given as functions of $|w|, c_{j}, d_{j}, s_{j}$, etc. with the help of two auxiliary functions $R(p, t, s)$ and $T(p, r, s)$ introduced and studied in [11: pp. 822 - 823] or, more precisely, [6: pp. $102-105]$ where $R(p, t, s)=r_{p}(t, s)$ and $T(p, r, s)=\tilde{\varrho}_{p}(r, s)$. They are deduced from the estimates for the classes $F_{\nu}$ of all mappings $f=g^{-1}, g \in$ $G_{\nu} \quad(\nu=1,2,3)$, that by $(1.4)$ satisfy

$$
e^{i \frac{2 \pi}{p}} f(z)=f\left(e^{i \frac{2 \pi}{p}} z\right)
$$

for all $z \in A_{\nu}$ with $A_{\nu}=g\left(B_{\nu}\right) \quad(\nu=1,2,3)$. Therefore the classes $F_{1}$ and $F_{2}$ introduced here are larger than $F_{1}$ and $F_{2}$ studied in [10], respectively, whose estimates will be partly improved. Our main tools are two inequalities due to Carleman [1: p. 212] and Grötzsch [2: p. 372] that were generalized and improved in [6 - 9] and especially in [10]. 


\section{Estimates for the classes $F_{1}$ and $G_{1}$}

To establish estimates for the class $G_{1}$ we need the following estimates for $F_{1}$.

Theorem 1. Under the above hypotheses and notations we have for every $f \in$ $F_{1}, 0<R<1,(0<) R_{j}(<1), j=1, \ldots$, pn and $(0 \neq) z \in A_{1}$

$$
\begin{aligned}
& (\pi \geq) S_{1}(f) \geq \pi S^{\prime}(0, f)+\sum_{j=1}^{p n} R_{j}^{-\frac{2}{K}} s_{j}(f) \\
& (\pi \geq) S(f) \geq \pi S^{\prime}(0, f)+\sum_{j=1}^{p n}\left(R_{j}^{-\frac{2}{K}}-1\right) s_{j}(f) \\
& (0 \leq) S^{\prime}(0, f) \leq \frac{S(f)}{\pi}(\leq 1) \\
& (0 \leq) p s_{j}(f) \leq\left[S_{1}(f)-\pi S^{\prime}(0, f)\right] R_{j}^{\frac{2}{K}} \\
& p s_{j}(f) \leq\left[S(f)-\pi S^{\prime}(0, f)\right]\left(R_{j}^{-\frac{2}{K}}-1\right)^{-1} \\
& (0 \leq) s(f) \leq\left[S_{1}(f)-\pi S^{\prime}(0, f)\right] R_{0}^{\frac{2}{K}} \\
& s(f) \leq\left[S(f)-\pi S^{\prime}(0, f)\right]\left(R_{0}^{-\frac{2}{K}}-1\right)^{-1} \\
& (0 \leq) s_{0}(f) \leq\left[S_{1}(f)-\pi S^{\prime}(0, f)\right]\left(\sum_{j=1}^{p n} R_{j}^{-\frac{2}{K}}\right)^{-1} \\
& s_{0}(f) \leq\left[S(f)-\pi S^{\prime}(0, f)\right]\left(\sum_{j=1}^{p n} R_{j}^{-\frac{2}{K}}-p n\right)^{-1} \\
& S^{\prime}(0, f) \pi R^{\frac{2}{K}} \leq S(R, f) \leq S_{1}(f) R^{\frac{2}{K}} \\
& m(R, f) \leq \sqrt{\frac{S_{1}(f)}{\pi}} R^{\frac{1}{K}} \\
& M(R, f) \geq \sqrt{S^{\prime}(0, f)} R^{\frac{1}{K}} \\
& m(R, f) \geq 4^{-\frac{1}{p}} m^{\prime}(0, f) R^{\frac{1}{K}}=m_{0}(\geq 0) \\
& M(R, f) \leq T\left(p, R^{\frac{1}{K}}, m_{0}\right) \leq T\left(p, R^{\frac{1}{K}}, 0\right)<4^{\frac{1}{p}} R^{\frac{1}{K}} \\
& m=4^{-\frac{1}{p}} m^{\prime}(0, f)|z|^{\frac{1}{K}} \leq|f(z)| \leq T\left(p,|z|^{\frac{1}{K}}, m\right)<4^{\frac{1}{p}}|z|^{\frac{1}{K}} \\
& m_{j}=4^{-\frac{1}{p}} m^{\prime}(0, f) R_{j}^{\frac{1}{K}} \leq c_{j}(f) \leq d_{j}(f) \leq T\left(p, R_{j}^{\frac{1}{K}}, m_{j}\right)<4^{\frac{1}{p}} R_{j}^{\frac{1}{K}} \\
& (1 \leq) \frac{d_{j}(f)}{c_{j}(f)}<2^{\frac{4}{p}} m^{\prime}(0, f)^{-1} \text { if } m^{\prime}(0, f)>0
\end{aligned}
$$

where equality in each of relations $(2.1)-(2.12)$ holds if and only if $f(z)=a z|z|^{\frac{1}{K}-1}$ with $|a|=1$.

Proof. Applying [10: Lemma 2.1] to the mapping $f \in F_{1}$ of the domain $A_{1}$ onto $B_{1}$, we have (2.1) and thus (2.2) since $S=S_{1}-s$. Because of the $p$-fold rotational symmetry (1.5) of $f \in F_{1}$ from (2.1) we obtain (2.4), (2.6) and (2.8). Similarly, from (2.2) we get (2.3), (2.5), (2.7) and (2.9). Applying again [10: Lemma 2.1] to the mapping $f \in F_{1}$ of the domain $A_{1} \cap\{|z|<R\}$ we obtain the lower estimate for $S(R, f)$ in (2.10), while the upper estimate holds by applying [9: Lemma 2.1] to the mapping $f \in F_{1}$ of the domain $A_{1} \cap\{|z|>R\}$. Combining this with the relation

$$
\pi m(R, f)^{2} \leq S(R, f) \leq \pi M(R, f)^{2}
$$

for $0<R<1$ and $f \in F_{1}$ yields (2.11) and (2.12). Equality in each of relations (2.1) - (2.12) holds if and only if $f(z)=a z|z|^{\frac{1}{K}-1}+b$, with $b=0$ since $f(0)=0$ and with $|a|=1$ since $M(1, f)=1$. The proof of estimates (2.13) - (2.17) is as in [10: p. 372] 
Remark 1. Theorem 1 with $S \leq S_{1} \leq \pi$ generalizes and improves [10: Theorem 2.1], where $C_{1}$ is the unit circle, i.e. $S=S_{1}=\pi$. It generalizes also [7: Theorem 3], where $K=1$.

Remark 2. The upper estimate for $|f(z)|$ in (2.15) presents a generalization of the Schwarz lemma to the case of quasiconformal mappings of finitely-connected domains. The sharpness of this estimate is open. In the particular case $n=0$ and $p=1$, where $A_{1}$ is the open unit disk, Hersch and Pfluger [3] showed the sharp upper estimate for $|f(z)|$ that under our notations has the form $|f(z)| \leq T\left(1, r^{\frac{1}{K}}, 0\right)$ with $r=R(1,|z|, 0), f \in F_{1}, z \in A_{1}$. Note that this cannot remain true for $n \geq 1$ by a similar example as in [9: pp. 62 - 63].

Corollary 1. For $K=1$ by $S^{\prime}(0, f)=\left|f^{\prime}(0)\right|^{2}$ from (2.3) we obtain

$$
\left|f^{\prime}(0)\right| \leq \sqrt{\frac{S(f)}{\pi}} \quad\left(f \in F_{1}\right)
$$

with equality if and only if $f(z)=a z$ with $|a|=1$.

By $S \leq \pi$ this improves the classical inequality $\left|f^{\prime}(0)\right| \leq 1$ for $f \in F_{1}$ with $K=1$ (see [5: p. 352]).

Lemma 1. Let $w=f(z)$ be a $K$-quasiconformal mapping of a domain containing $z=0$ with $f(0)=0$ and $m^{\prime}(0, f)>0$. Then for $g=f^{-1}$ we have

$$
\begin{aligned}
& m^{\prime}(0, f)=M^{*}(0, g)^{-\frac{1}{K}} \\
& M^{\prime}(0, f)=m^{*}(0, g)^{-\frac{1}{K}} .
\end{aligned}
$$

Proof. For small $R>0$ put $C_{R}=\{z:|z|=R\}$ and $C_{R}^{\prime}=f\left(C_{R}\right)$. Clearly, there exist a point $w_{1} \in C_{R}^{\prime}$ and a point $z_{1} \in C_{R}$ such that

$$
m(R, f)=\left|w_{1}\right|=\left|f\left(z_{1}\right)\right|=r .
$$

Put $L_{r}=\{w:|w|=r\}$ and $L_{r}^{\prime}=g\left(L_{r}\right)$. Noticing that $L_{r}^{\prime}$ is situated in $|z| \leq R$, we get

$$
M(r, g)=\left|g\left(w_{1}\right)\right|=\left|z_{1}\right|=R .
$$

Thus, since $m^{\prime}(0, f)>0$ we conclude

$$
m^{\prime}(0, f)=\lim _{R \rightarrow 0} \frac{m(R, f)}{R^{\frac{1}{K}}}=\lim _{r \rightarrow 0} \frac{r}{M(r, g)^{\frac{1}{K}}}=\lim _{r \rightarrow 0}\left[\frac{M(r, g)}{r^{K}}\right]^{-\frac{1}{K}}=M^{*}(0, g)^{-\frac{1}{K}} .
$$

Similarly we can prove $(2.21)$

Remark 3. For $K=1$, since $m^{\prime}(0, f)=\left|f^{\prime}(0)\right|$ and $M^{*}(0, g)=\left|g^{\prime}(0)\right|$, equality (2.20) becomes the well-known relation $\left|f^{\prime}(0)\right|=\left|g^{\prime}(0)\right|^{-1}$. 
Theorem 2. Under the above hypotheses and notations we have for every $g \in$ $G_{1}, w \in B_{1}$ and $j=1, \ldots, p n$

$$
\begin{aligned}
& M^{*}(0, g) \geq\left(\frac{\pi}{S}\right)^{\frac{K}{2}}(\geq 1) \\
& M^{*}(0, g)>2^{-\frac{4 K}{p}}\left(\frac{d_{j}}{c_{j}}\right)^{K} \\
& R_{j}(g)>\left[\frac{p s_{j}}{p s_{j}+S-\pi M^{*}(0, g)^{-\frac{2}{K}}}\right]^{\frac{K}{2}} \text { with } s_{j}>0 \\
& R_{0}(g)>\left[\frac{s}{S_{1}-\pi M^{*}(0, g)^{-\frac{2}{K}}}\right]^{\frac{K}{2}} \text { with } s>0 \\
& (p n<) \sum_{j=1}^{p n} R_{j}^{-\frac{2}{K}}(g)<\frac{S_{1}-\pi M^{*}(0, g)^{-\frac{2}{K}}}{s_{0}} \text { with } s_{0}>0 \\
& \sum_{j=1}^{p n} R_{j}^{-\frac{2}{K}}(g)<p n+\frac{S-\pi M^{*}(0, g)^{-\frac{2}{K}}}{s_{0}} \text { with } s_{0}>0 \\
& 4^{-\frac{K}{p}} d_{j}^{K}<R\left(p, d_{j}, 0\right)^{K} \leq R_{j}(g) \leq 4^{\frac{K}{p}} M^{*}(0, g) c_{j}^{K} \\
& R_{j}(g)>\left[\frac{p s_{j}}{Q_{j}(g)}\right]^{\frac{K}{2}}
\end{aligned}
$$

with $s_{j}>0$ and $Q_{j}(g)=S_{1}-\left(\pi+2^{-\frac{4}{p}} \sum_{R_{\nu} \neq R_{j}} \frac{s_{\nu}}{c_{\nu}^{2}}\right) M^{*}(0, g)^{-\frac{2}{K}}(>0)$ and

$$
4^{-\frac{K}{p}}|w|^{K}<R(p,|w|, 0)^{K} \leq|g(w)| \leq 4^{\frac{K}{p}} M^{*}(0, g)|w|^{K}
$$

Equality in relation (2.22) holds if and only if $B_{1}=B_{1}^{0}$, where $B_{1}^{0}$ means the open unit disk that has been slit along pn circular arcs concentric with the unit circle, and $g(w)=a w|w|^{K-1}$ with $|a|=1$.

Proof. Combining (1.1), (2.3) and (2.20) yields estimate (2.22) with equality if and only if

$$
w=f(z)=g^{-1}(z)=b z|z|^{\frac{1}{K}-1} \quad \text { with }|b|=1 .
$$

This implies the above assertion in the case of equality in (2.22). Similarly, from (2.17) estimate (2.23) follows. With the help of (1.1) and (2.20), by (2.5), (2.6), (2.8) and (2.9) we obtain inequalities (2.24) - (2.27), respectively. From (2.16) we get

$$
d_{j} \leq T\left(p, R_{j}^{\frac{1}{K}}, m_{j}\right) \leq T\left(p, R_{j}^{\frac{1}{K}}, 0\right)=t_{j} .
$$

Thus, by the definitions of the auxyliary functions $T(p, r, s)$ and $R(p, t, s)$ and their monotonys (see [11: pp. $822-823]$ ) we conclude

$$
R_{j}^{\frac{1}{K}}=R\left(p, t_{j}, 0\right) \geq R\left(p, d_{j}, 0\right)>4^{-\frac{1}{p}} d_{j},
$$

hence the lower estimate for $R_{j}$ in (2.28) follows, while its upper estimate is deduced easily from (2.16) and (2.20). Writing (2.1) by (1.5) in the form

$$
S_{1} \geq \pi S^{\prime}(0, f)+\frac{p s_{j}}{R_{j}^{\frac{2}{K}}}+\sum_{R_{\nu} \neq R_{j}} \frac{s_{\nu}}{R_{\nu}^{\frac{2}{K}}}
$$

and using (1.1), (2.20) and the upper estimate for $R_{\nu}$ in (2.28) we obtain (2.29). Estimate (2.30) is deduced from (2.15) and (2.20) similarly as in the proof of (2.28) 
Corollary 2. For $K=1$ estimate (2.22) becomes

$$
\left|g^{\prime}(0)\right| \geq \sqrt{\frac{\pi}{S}} \quad\left(g \in G_{1}\right)
$$

with equality if and only if $B_{1}=B_{1}^{0}$ and $g(w)=a w$ with $|a|=1$.

Estimate (2.31) with $S \leq \pi$ improves the classical inequality $\left|g^{\prime}(0)\right| \geq 1$ for $g \in G_{1}$ with $K=1$.

In order to establish an estimate that can sharpen (2.22) and therefore (2.31) we shall prove

Corollary 3. Putting $C=2^{-\frac{4}{p}} \sum_{j=1}^{p n} \frac{s_{j}}{c_{j}^{2}}(\geq 0)$, for every $g \in G_{1}$ we have

$$
M^{*}(0, g) \geq\left(\frac{\pi+C}{S_{1}}\right)^{\frac{K}{2}}
$$

with equality if and only if $B_{1}=B_{1}^{0}$ and $g(w)=a w|w|^{K-1}$ with $|a|=1$.

Proof. Combining (1.1), (2.1), (2.20) and (2.28) yields

$$
S_{1} \geq \pi M^{*}(0, g)^{-\frac{2}{K}}+C M^{*}(0, g)^{-\frac{2}{K}}
$$

hence (2.32) follows with the above assertion in the case of equality

Corollary 4. In the case $K=1$, where $M^{*}(0, g)=\left|g^{\prime}(0)\right|$, estimate (2.32) becomes

$$
\left|g^{\prime}(0)\right| \geq \sqrt{\frac{\pi+C}{S_{1}}} \quad\left(g \in G_{1}\right)
$$

with equality if and only if $B_{1}=B_{1}^{0}$ and $g(w)=a w$ with $|a|=1$.

\section{Estimates for the classes $\boldsymbol{F}_{2}$ and $\boldsymbol{G}_{2}$}

To establish estimates for the class $G_{2}$ we need the following estimates for $F_{2}$.

Theorem 3. Under the hypotheses and notations given in Section 1, for $f \in$ $F_{2}, z \in A_{2}, 1<R<\infty,(1<) R_{j}(<\infty) \quad(j=1, \ldots, p n)$ we have the estimates

$$
\begin{aligned}
S^{\prime}(\infty, f) & \geq \frac{S_{2}(f)}{\pi}+\sum_{j=1}^{p n} \frac{s_{j}(f)}{\pi R_{j}^{\frac{2}{K}}}\left(\geq \frac{S_{2}}{\pi} \geq 1\right) \\
p s_{j}(f) & \leq\left[\pi S^{\prime}(\infty, f)-S_{2}(f)\right] R_{j}^{\frac{2}{K}} \\
s_{0}(f) & \leq\left[\pi S^{\prime}(\infty, f)-S_{2}(f)\right]\left(\sum_{j=1}^{p n} R_{j}^{-\frac{2}{K}}\right)^{-1} \\
s(f) & \leq\left[\pi S^{\prime}(\infty, f)-S_{2}(f)\right] R_{0}^{\frac{2}{K}} \\
\left(\pi R^{\frac{2}{K}} \leq\right) S_{2}(f) R^{\frac{2}{K}} & \leq S(R, f) \leq S^{\prime}(\infty, f) \pi R^{\frac{2}{K}} \\
M(R, f) & \geq \sqrt{\frac{S_{2}(f)}{\pi}} R^{\frac{1}{K}}
\end{aligned}
$$




$$
\begin{aligned}
& m(R, f) \leq \sqrt{S^{\prime}(\infty, f)} R^{\frac{1}{K}} \\
& M(R, f)<4^{\frac{1}{p}} M^{\prime}(\infty, f) R^{\frac{1}{K}}=M_{0} \\
& m(R, f) \geq T\left(p, R^{-\frac{1}{K}}, M_{0}^{-1}\right)^{-1} \geq T\left(p, R^{-\frac{1}{K}}, 0\right)^{-1}>4^{-\frac{1}{p}} R^{\frac{1}{K}} \\
& 4^{-\frac{1}{p}}|z|^{\frac{1}{K}}<T\left(p,|z|^{-\frac{1}{K}}, M^{-1}\right)^{-1} \leq|f(z)|<4^{\frac{1}{p}} M^{\prime}(\infty, f)|z|^{\frac{1}{K}}=M \\
& 4^{-\frac{1}{p}} R_{j}^{\frac{1}{K}}<T\left(p, R_{j}^{-\frac{1}{K}}, M_{j}^{-1}\right)^{-1} \leq c_{j} \leq d_{j}<4^{\frac{1}{p}} M^{\prime}(\infty, f) R_{j}^{\frac{1}{K}}=M_{j} \\
& (1 \leq) \frac{d_{j}}{c_{j}}<2^{\frac{4}{p}} M^{\prime}(\infty, f)
\end{aligned}
$$

where equality in each of relations $(3.1)-(3.7)$ holds if and only if $f(z)=a z|z|^{\frac{1}{K}-1}$ with $|a|=1$.

Proof. Applying [10: Lemma 3.1] to the mapping $f \in F_{2}$ of the domain $A_{2}$ onto $B_{2}$, we have (3.1) and therefore (3.2) - (3.4). Applying again this lemma to the mapping $f \in F_{2}$ of the domain $A_{2} \cap\{|z|>R\}$, we get the upper estimate for $S(R, f)$ in (3.5), while the lower estimate holds by applying [9: Lemma 2.1] to the mapping $f \in F_{2}$ of the domain $A_{2} \cap\{|z|<R\}$. Thus, by (2.18) for $R>1$ and $f \in F_{2}$, we obtain estimates (3.6) and (3.7). The equality in each of relations (3.1) - (3.7) holds if and only if $f(z)=a z|z|^{\frac{1}{K}-1}+b$ with $b=0$ and $|a|=1$ because of the conditions of $C_{2}$. The proof of estimates (3.8) - (3.12) is as in [10: pp. $\left.374-375\right]$

Remark 4. Theorem 3 with $S_{2} \geq \pi$ generalizes and improves [10: Theorem 3.1], where $C_{2}$ is the circle $|w|=1$, i.e. $S_{2}=\pi$. It generalizes also [7: Theorem 5], where $K=1$.

Lemma 2. Let $w=f(z)$ be a $K$-quasiconformal mapping of a domain containing $z=\infty$ with $f(\infty)=\infty$ and $M^{\prime}(\infty, f)>0$. Then for $g=f^{-1}$ we have

$$
\begin{aligned}
& M^{\prime}(\infty, f)=m^{*}(\infty, g)^{-\frac{1}{K}} \\
& m^{\prime}(\infty, f)=M^{*}(\infty, g)^{-\frac{1}{K}} .
\end{aligned}
$$

Proof. Similarly to the proof of Lemma 1, we can prove this lemma

Theorem 4. Under the hypotheses and notations given in Section 1, for $g \in$ $G_{3}, w \in B_{3}$ and $j=1, \ldots$, pn we have the estimates

$$
\begin{aligned}
(0 \leq) m^{*}(\infty, g) & \leq\left(\frac{\pi}{S_{2}}\right)^{\frac{K}{2}}(\leq 1) \\
m^{*}(\infty, g) & <2^{\frac{4 K}{p}}\left(\frac{c_{j}}{d_{j}}\right)^{K} \\
4^{-\frac{K}{p}} m^{*}(\infty, g) d_{j}^{K} & \leq R_{j}(g) \leq R\left(p, c_{j}^{-1}, 0\right)^{-K}<4^{\frac{K}{p}} c_{j}^{K} \\
R_{j}(g) & >\left[\frac{p s_{j}}{V_{j}(g)}\right]^{\frac{K}{2}}
\end{aligned}
$$

with $s_{j}>0$ and

$$
\begin{aligned}
(0<) V_{j}(g) & =\pi m^{*}(\infty, g)^{-\frac{2}{K}}-S_{2}-\sum_{R_{\nu} \neq R_{j}} s_{\nu} R\left(p, c_{\nu}^{-1}, 0\right)^{2} \\
& \leq \pi m^{*}(\infty, g)^{-\frac{2}{K}}-S_{2}-2^{-\frac{4}{p}} \sum_{R_{\nu} \neq R_{j}} \frac{s_{\nu}}{c_{\nu}^{2}}
\end{aligned}
$$


and

$$
\begin{aligned}
R_{0}(g) & >\left[\frac{s}{\pi m^{*}(\infty, g)^{-\frac{2}{K}}-S_{2}}\right]^{\frac{K}{2}} \text { with } s>0 \\
\sum_{j=1}^{p n} R_{j}^{-\frac{2}{K}}(g) & <\frac{\pi m^{*}(\infty, g)^{-\frac{2}{K}}-S_{2}}{s_{0}} \text { with } s_{0}>0 \\
4^{-\frac{K}{p}} m^{*}(\infty, g)|w|^{K} & <|g(w)|<R\left(p,|w|^{-1}, 0\right)<4^{\frac{K}{p}}|w|^{K}
\end{aligned}
$$

with equality in (3.14) if and only if $B_{2}=B_{2}^{0}$, where $B_{2}^{0}$ means the domain $|w|>$ 1 that has been slit along pn circular arcs concentric with $|w|=1$, and $g(w)=$ $a w|w|^{K-1}$ with $|a|=1$. if

Proof. Combining (1.1), (3.1) and (3.13) yields (3.14) with equality if and only

$$
w=f(z)=g^{-1}(z)=b z|z|^{\frac{1}{K}-1} \quad \text { with }|b|=1 .
$$

This implies the above assertion in the case of equality in (3.14). Estimate (3.15) follows from (3.12) and (3.13). By the definitions of the auxiliary functions $T(p, r, s)$ and $R(p, t, s)$ and their monotony (see [11: p. 822]) we get from (3.11) the equivalence

$$
c_{j}^{-1} \leq T\left(p, R_{j}^{-\frac{1}{K}}, 0\right)=t_{j} \quad \Longleftrightarrow \quad R_{j}^{-\frac{1}{K}}=R\left(p, t_{j}, 0\right) \geq R\left(p, c_{j}^{-1}, 0\right),
$$

hence the upper estimate for $R_{j}(g)$ in (3.16) follows, while the lower estimate is deduced easily from (3.11) and (3.13). By (1.1), (1.5) and (3.13) relation (3.1) can be represented in the form

$$
m^{*}(\infty, g)^{-\frac{2}{K}} \geq \frac{S_{2}}{\pi}+\frac{p s_{j}}{\pi R_{j}^{\frac{2}{K}}}+\sum_{R_{\nu} \neq R_{j}} \frac{s_{\nu}}{\pi R_{\nu}^{\frac{2}{K}}} .
$$

Thus, using the upper estimate in (3.16) for $R_{\nu}$, we get (3.17). With the help of (3.13) relations (3.18) and (3.19) are deduced from (3.4) and (3.3), respectively. Similarly to the proof of (3.16), using (3.10) and (3.13) we can show (3.20)

In order to improve estimate (3.14) we shall prove

\section{Corollary 5. Putting}

$$
D=\sum_{j=1}^{p n} s_{j} R\left(p, c_{j}^{-1}, 0\right)^{2} \geq 2^{-\frac{4}{p}} \sum_{j=1}^{p n} \frac{s_{j}}{c_{j}^{2}}(\geq 0)
$$

we have for every $g \in G_{2}$

$$
m^{*}(\infty, g) \leq\left(\frac{\pi}{S_{2}+D}\right)^{\frac{K}{2}} \leq\left(\frac{\pi}{S_{2}}\right)^{\frac{K}{2}}(\leq 1)
$$

with equality if and only if $B_{2}=B_{2}^{0}$ and $g(w)=a w|w|^{K-1}$ with $|a|=1$.

Proof. Combining (1.1), (3.1), (3.13) and (3.16) yields $m^{*}(\infty, g)^{-\frac{2}{K}} \geq \frac{S_{2}+D}{\pi}$, hence $(3.21)$ with the above assertion in the case of equality 
Corollary 6. In the case $K=1$, where

$$
m^{*}(\infty, g)=\lim _{R \rightarrow \infty} \frac{m(R, g)}{R}=\lim _{z \rightarrow \infty} \frac{|g(z)|}{|z|}=\left|g^{\prime}(\infty)\right|
$$

inequality (3.21) becomes

$$
\left|g^{\prime}(\infty)\right| \leq \sqrt{\frac{\pi}{S_{2}+D}} \quad\left(g \in G_{2}\right)
$$

with equality if and only if $B_{2}=B_{2}^{0}$ and $g(w)=a w$ with $|a|=1$.

This sharpens the classical inequality $\left|g^{\prime}(\infty)\right| \leq 1$ for $g \in G_{2}$ with $K=1$.

\section{Estimates for the class $G_{3}$}

Since estimates for the class $F_{3}$ with $M^{\prime}(\infty, f)=m^{*}(\infty, g)^{-\frac{1}{K}}=1$ for $g^{-1}=f \in F_{3}$ by (3.13) were shown in [10] we can now establish them for the class $G_{3}$.

Theorem 5. Under the hypotheses and notations given in Section 1 we have for every $g \in G_{3}, w \in B_{3}$ and $j=1, \ldots$, pn the estimates

$$
\begin{aligned}
M^{*}(0, g) & \geq 1 \\
M^{*}(0, g) & \geq 2^{-\frac{4 K}{p}}\left(\frac{d_{j}}{c_{j}}\right)^{K} \\
4^{-\frac{K}{p}} d_{j}^{K} & \leq R_{j}(g) \leq 4^{\frac{K}{p}} M^{*}(0, g) c_{j}^{K} \\
R_{j}(g) & >\left[\frac{p s_{j}}{T_{j}(g)}\right]^{\frac{K}{2}}>\left(\frac{p s_{j}}{\pi}\right)^{\frac{K}{2}}
\end{aligned}
$$

with $s_{j}>0$ and $T_{j}(g)=\pi-\left(\pi+2^{-\frac{4}{p}} \sum_{R_{\nu} \neq R_{j}} \frac{s_{\nu}}{c_{\nu}^{2}}\right) M^{*}(0, g)^{-\frac{2}{K}}(>0)$ and

$$
\begin{aligned}
R_{0}(g) & >\left\{\frac{s}{\pi\left[1-M^{*}(0, g)^{-\frac{2}{K}}\right]}\right\}^{\frac{K}{2}} \text { with } s>0 \\
\sum_{j=1}^{p n} R_{j}^{-\frac{2}{K}}(g) & \leq \frac{\pi}{s_{0}}\left[1-M^{*}(0, g)^{-\frac{2}{K}}\right] \text { with } s_{0}>0 \\
4^{-\frac{K}{p}}|w|^{K} & \leq|g(w)| \leq 4^{\frac{K}{p}} M^{*}(0, g)|w|^{K}
\end{aligned}
$$

with equality in (4.1) if and only if $B_{3}=B_{3}^{0}$, where $B_{3}^{0}$ means the extended $w$ plane that has been slit along pn circular arcs concentric with $|w|=1$, and $g(w)=$ $a w|w|^{K-1}$ with $|a|=1$.

Proof. Applying [10: Lemma 4.1] to the mapping $f \in F_{3}$ of $A_{3}$ onto $B_{3}$, we obtain by (1.1) and (2.20) for $g \in G_{3}$

$$
\sum_{j=1}^{p n} \frac{s_{j}}{\pi R_{j}^{\frac{2}{K}}(g)} \leq 1-M^{*}(0, g)^{-\frac{2}{K}}
$$


hence (4.1) with the above assertion in the case of equality. Estimate (4.2) follows from [10: Formula 4.13] and (2.20), while (4.3) is deduced from [10: Formula 4.12] and (2.20). By the $p$-fold rotational symmetry of $g \in G_{3}$ inequality (4.8) can be written in the form

$$
\frac{p s_{j}}{\pi R_{j}^{\frac{2}{K}}}+\sum_{R_{\nu} \neq R_{j}} \frac{s_{\nu}}{\pi R_{\nu}^{\frac{2}{K}}} \leq 1-M^{*}(0, g)^{-\frac{2}{K}},
$$

hence using upper estimate (4.3) for $R_{\nu}$, we get (4.4). Estimates (4.5) and (4.6) follow from (4.8). Combining [10: Corollary 4.1] with (2.20) yields (4.7)

In order to improve estimate (4.1) we shall prove

Corollary 7. Putting

$$
E=\left(\pi 2^{\frac{4}{p}}\right)^{-1} \sum_{j=1}^{p n} \frac{s_{j}}{c_{j}^{2}}(\geq 0)
$$

we have for every $g \in G_{3}$

$$
M^{*}(0, g) \geq(1+E)^{\frac{K}{2}}
$$

with equality if and only if $B_{3}=B_{3}^{0}$ and $g(w)=a w|w|^{K-1}$ with $|a|=1$.

Proof. Combining (4.8) with (4.3) yields (4.9) with the above assertion in the case of equality

Corollary 8. In the case $K=1$, where $M^{*}(0, g)=\left|g^{\prime}(0)\right|$, estimate (4.9) becomes

$$
\left|g^{\prime}(0)\right| \geq \sqrt{1+E} \quad\left(g \in G_{3}\right)
$$

with equality if and only if $B_{3}=B_{3}^{0}$ and $g(w)=a w$ with $|a|=1$.

This sharpens the classical inequality $\left|g^{\prime}(0)\right| \geq 1$ for $g \in G_{3}$ with $K=1$ (see [5: p. 350]).

Concluding Remark. All estimates obtained in this paper are sharp or asymptotically sharp. This follows from [10: p. 377].

Acknowledgement. This paper has been prepared during the research visit of the author at the FU Berlin in the fourth quarter of 2001 under the patronage of DAAD. I would like to thank the DAAD, Professor Heinrich Begehr and his group for their kind support. 


\section{References}

[1] Carleman, T.: Über ein Minimalproblem der mathematischen Physik. Math. Z. 1 (1918), $208-212$.

[2] Grötzsch, H.: Über einige Extremalprobleme der konformen Abbildung. Ber. Verhandl. Sächs. Akad. Wiss. Leipzig, math.-phys. Klasse 80 (1928), $367-376$.

[3] Hersch, J. and A. Pfluger: Généralization du lemme de Schwarz et du principe de la mesure harmonique pour les fonctions pseudo-analytiques. C.R. Acad. Sci. Paris 234 (1952), $43-45$.

[4] Lehto, O. and K. I. Virtanen: Quasikonforme Abbildungen. Berlin - Heidelberg - New York: Springer 1965.

[5] Nehari, Z.: Conformal Mapping. New York: McGraw-Hill Book Comp. 1952.

[6] Thao, V. D.: Verhalten schlicht-konformer Abbildungen in Kreisringe eingebetteter Gebiete. Math. Nachr. 74 (1976), 99 - 134.

[7] Thao, V. D.: Über einige Flächeninhaltsformeln bei schlichtkonformer Abbildung von Kreisbogenschlitzgebieten. Math. Nachr. 74 (1976), $253-261$.

[8] Thao, V. D.: Quelques inégalités d'aires pour les représentations quasi-conformes. Rev. Roum. Math. Pures Appl. 36 (1991), 521 - 527.

[9] Thao, V. D.: Estimations pour les représentations quasi-conformes des domaines plans, Part I. Rev. Roum. Math. Pures Appl. 38 (1993), 55 - 66.

[10] Thao, V. D.: Estimations pour les représentations quasi-conformes des domaines plans, Part II. Rev. Roum. Math. Pures Appl. 38 (1993), 369 - 378.

[11] Thao, V. D.: Estimates for quasiconformal mappings onto canonical domains. Z. Anal. Anw. 18 (1999), $819-825$.

Received 09.01.2002 